\section{PSICOLOGIA IBEROAMERICANA}

\section{Psicología lberoamericana}

ISSN: 1405-0943

psicología.iberoamericana@uia.mx

Universidad Iberoamericana, Ciudad de

México

México

López Mejía, David Iñaki; Valdovinos de Yahya, Azucena; Méndez-Díaz, Mónica; Mendoza-

Fernández, Víctor

El Sistema Límbico y las Emociones: Empatía en Humanos y Primates

Psicología Iberoamericana, vol. 17, núm. 2, julio-diciembre, 2009, pp. 60-69

Universidad Iberoamericana, Ciudad de México

Distrito Federal, México

Disponible en: http://www.redalyc.org/articulo.oa?id=133912609008

Cómo citar el artículo

- Número completo

- Más información del artículo

- Página de la revista en redalyc.org

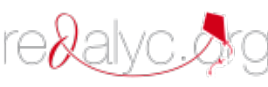

Sistema de Información Científica

Red de Revistas Científicas de América Latina, el Caribe, España y Portugal

Proyecto académico sin fines de lucro, desarrollado bajo la iniciativa de acceso abierto 


\title{
El Sistema Límbico y las Emociones: Empatía en Humanos y Primates
}

\author{
Limbic System and Emotions: Empathy in Humans and Primates
}

\author{
David Iñaki López Mejía* Azucena Valdovinos de Yahya** \\ Mónica Méndez-Díaz* , Víctor Mendoza-Fernández***1 \\ *Departamento de Psicología, Universidad Iberoamericana, CD. de México, México \\ * * Colaboradora de la Revista Pshychologies, México \\ ** *Grupo de Neurociencias, Departamento de Fisiología, Facultad de Medicina, unam, Cd. de México, México
}

\section{RESUMEN}

El sistema límbico es un conjunto de estructuras cerebrales que responden a ciertos estímulos ambientales produciendo respuestas emocionales; como: miedo, alegría, enojo o tristeza. Aunque dichas emociones han sido consideradas características únicas del humano, Chales Darwin las describió en varias especies y en animales cercanos en la escala filogenética del humano, como los primates.

A principios del siglo XIX se describió el mecanismo por el cual este sistema regula las emociones y cómo los seres humanos reconocen y comparten las mismas (empatía). La descripción de los generadores de patrones centrales (CPG’s), como estructuras anatómico-funcionales conservadas evolutivamente, sugiere su participación en la regulación de varias actividades, incluidas las emociones y la empatía. Lo que daría un carácter de universalidad e incluso de reconocimiento conductual inter especies. En el presente trabajo se relaciona al sistema límbico y a los CPG's como estructuras involucradas en la empatía en los humanos y primates.

Descriptores: Sistema Límbico, Emociones, Empatía, Evolución, Neuropsicología de las emociones, CPG.

\section{ABSTRACT}

The limbic system is a group of brain structures that react to certain external stimulus inducing emotional behaviors like: fear, happiness, angriness and sadness. This has been considered for long time as a unique characteristic of humans; however, Charles Darwin described this emotions in several species and in primates, animals phylogenetic related to humans.

At the beginning of the XIX century the mechanism used by this system to regulate the human emotional responses and how the humans recognize and share them (empathy) was described. The description of the central patterns generators (CPG's) as anatomic-functional structures evolutively conserved, suggest its participation on several neuronal activities regulation including emotional and empathic activities. This gives to the empathy universality characteristic and inter-spices behaviors recognize. In this paper the limbic system and CPG's relation is propose as a structures involve in human and primates empathy.

Keywords: Limbic System, Emotions, Empathy, Evolution, Neuropsychology CPG's.

\footnotetext{
${ }^{1}$ Para Correspondencia: David Iñaki López Mejía, Edificio J, Nivel 2, Universidad Iberoamericana, Prolongación Paseo de la Reforma 880, Lomas de Santa Fe, C.P. 01219, México D.F. Tel.: (52) (55) 5364 1209, cel.: (044) 5514879645, iñaki.lopez.m@gmail.com. Azucena Valdovinos de Yahya Colaboradora de la Revista Pshychologies, Av. Bernardo Quintana 595 casa 29A, La Loma, Santa Fe, C.P. 01210, México, D.F. Cel.: (044) 5554042247, azucena.valdovinos@gmail.com. Dra. Mónica Méndez Díaz, Departamento de Fisiología, Facultad de Medicina, Edificio A, Laboratorio 601. Ciudad Universitaria, Av. Universidad 3000. APDO. POSTAL 70-450, C.P. 04510, México, D.F. Tel.: (52) (55) 5623 2509, mendezm@unam.mx. Dr. Víctor Mendoza-Fernández, Departamento de Psicología, Edificio J, Nivel 2, Universidad Iberoamericana, Prolongación Paseo de la Reforma 880, Lomas de Santa Fe, C.P. 01219, México, D.F. Tel. (52) (55) 5648-0423, cel.: (044) 55 91930879, mefevic@yahoo.com, mendozav@ymail.com
} 


\section{INTRODUCCIÓN}

En el amplio espectro de la conducta del humano, las emociones han representado un tema interesante para el análisis, en primer lugar debido a que controlan conductas complejas en el humano como la motivación y el aprendizaje (Purves, 2004), y en segundo lugar porque la mayoría de las enfermedades psiquiátricas más devastadoras (por ejemplo: depresión, esquizofrenia y trastornos afectivos) involucran desordenes emocionales (Kandel, 2000). Las respuestas emocionales que conocemos en los humanos son una variedad de felicidad, sorpresa, enojo, miedo y tristeza. Todas ellas presentan dos características comunes: la primera se refiere a una respuesta motora visceral y la segunda a una respuesta motora estereotipada somática. Con base en los objetivos de este artículo, nos enfocaremos a describir la segunda característica. La respuesta somática involucra principalmente movimiento de los músculos faciales, acompañada de elementos subjetivos difíciles de describir, pero que se encuentran preservados universalmente en las diferentes culturas, y que por lo tanto nos permiten identificar las emociones de los demás. La sensibilidad que presenta una persona hacia las emociones de otra y la sensibilidad para entender la dinámica de interacción con esa segunda persona es llamada empatía. La empatía es la capacidad cognitiva que tiene una persona para sentir lo que siente otra; ello puede llevar a una mejor comprensión de su comportamiento o forma de tomar decisiones. Se trata de la habilidad para entender las necesidades, sentimientos y problemas de los demás, para ponerse en su lugar y responder correctamente ante sus reacciones emocionales. La empatía incluye el conocimiento y el uso de las emociones para comprender a las personas, el mundo que nos rodea e incluso la naturaleza (Lovecky, 2004).

El conjunto de núcleos cerebrales que regulan las emociones forman el Sistema Límbico (área ventral tegmental, núcleo accumbens, hipocampo, núcleos septales laterales, corteza frontal). Recientemente, otras estructuras han sido adicionadas al sistema límbico tradicional (Gelder, Morris \& Dolan, 2005). Éstas son la amígdala y la corteza orbito-frontal. El sistema límbico junto con las estructuras de la corteza frontal, procesan los estímulos emocionales y los integran a funciones cerebrales complejas, las cuales incluyen: decisiones racionales, expresión e interpretación de conductas sociales e incluso la generación de juicios morales, entendiéndose estos últimos como los actos mentales que afirman o niegan el valor moral frente a una situación o comportamiento (Kandel, 2000).

El desarrollo antropológico del sistema límbico, la amígdala y las áreas corticales orbito-frontales está estrechamente relacionado con el desarrollo general del cerebro a los largo de la escala filogenética del ser humano. Incluso este patrón se conserva con ciertas consideraciones en otros animales, como los primates (Barton, Aggleton \& Grenyer, 2003; Randall, 1984). En específico, los primates podrían generar empatía debido principalmente a las coincidencias neuroanatómicas que presentan con el ser humano. En los animales, el desarrollo del lóbulo frontal está limitado por un proceso de neurogénesis regulado por factores intraneuronales (Heltne \& Marquardt, 1989). Lo anterior representa un hecho fundamental para los procesos de cognición y raciocinio, los cuales representan los pasos esenciales en la generación de empatía (Figura 1).

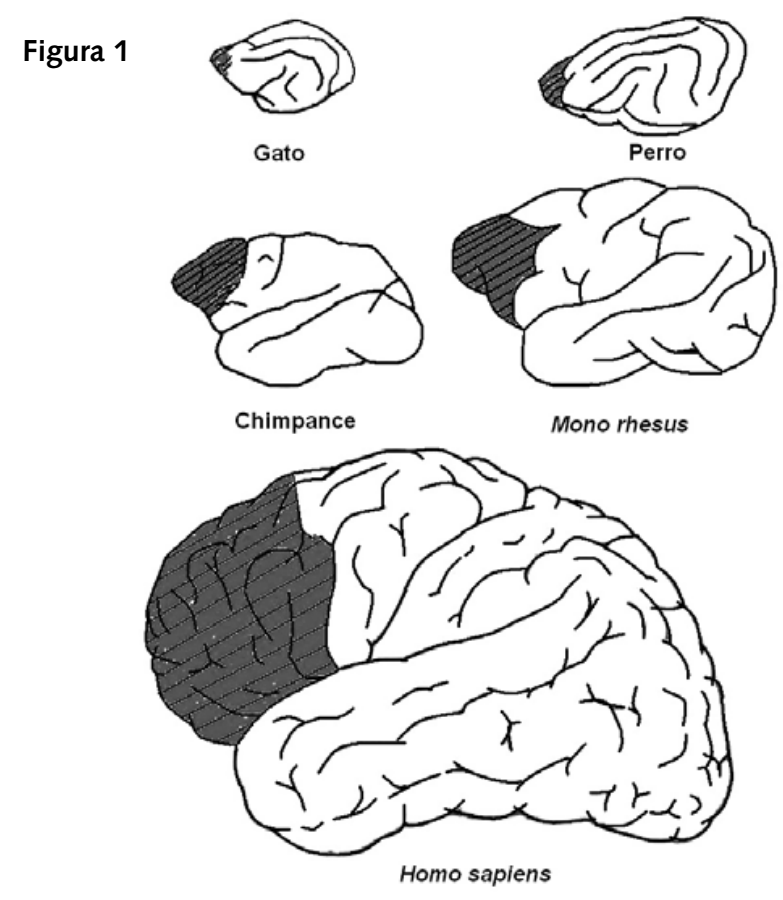

Nota: Dibujo que muestra las diferencias filogenéticas en el tamaño del cerebro de cinco especies de mamíferos, incluyendo al hombre (Homo sapiens). En amarillo se señala el área de la corteza que ocupa en cada especie la corteza frontal. Se ha sugerido que esta diferencia está relacionada con las variaciones en las conductas que presentan cada especie. 
Además, se ha descrito también la existencia de grupos neuronales denominados Generadores de $\mathrm{Pa}$ trones Centrales (CPG, por sus siglas en inglés para Central Patterns Generator) localizados en el mescencéfalo, puente y medula espinal, tanto en humanos como en animales, los cuales permiten la preservación inter-especie de respuestas motoras, entre las que se encuentran las generadas por las emociones (al mover los músculos faciales) y por ende la posibilidad de compartir la conducta de empatía (Briggman \& Kristan, 2006; Korb, Grandjean \& Scherer, 2008). Los CPG's son cadenas neuronales capaces de generar patrones rítmicos de actividad motora, activados principalmente por la estimulación de los receptores sensoriales periféricos y por señales generadas por otros núcleos del sistema nervioso central.

La existencia de rasgos de empatía en los animales y para la conservación de éstos en diferentes razas y culturas en los humanos, puede ser la existencia de CPG's conservados en las escala filogenética de los mamíferos. De esta forma, el sistema límbico formaría parte de uno o varios CPG's que controlan la respuesta emocional y la empatía en los humanos. Probablemente esta misma relación está conservada en los primates. Hay que tomar en cuenta que, por una parte el sistema límbico regula la expresión de las respuestas emocionales y, por otra, los CPG's asociados a este sistema inician y controlan la actividad de los músculos faciales, generando una respuesta estereotipada y conservadora ante un estímulo específico (por ejemplo cuando se emite una emoción). Esta información nos permitiría comprender desde un punto de vista neurofisiológico y neuroanatómico las descripciones realizadas por Charles Darwin hace ya más de 125 años en su libro La expresión de las emociones en los humanos y en los animales (Darwin, 1998).

\section{EL SISTEMA LÍMBICO HUMANO Y DE LOS ANIMALES}

El placer, la tristeza, la depresión, el miedo, el enojo, la hostilidad, la ansiedad, son emociones que dan ciertas tonalidades a nuestra vida cotidiana, que enriquecen cada una de nuestras experiencias y nos permiten aplicar el conocimiento obtenido con pasión y carácter. Cuando la intensidad y características de estas emociones salen de los valores "normales" o fisiológicos, se presentan desórdenes emocionales, los cuales constituyen un gran número de enfermedades mentales, por ejemplo la depresión, las psicosis y los trastornos de afectividad.

Según E. Kandel (2000), el estado emocional de los humanos está compuesto por un elemento evidente caracterizado principalmente por las sensaciones físicas y otro caracterizado por un sentimiento concreto (por ejemplo, cuando se tiene la sensación de "pesadez" en el corazón, concretamente se siente miedo: es decir, se interpreta una sensación). En resumen, para mantener una distinción semántica entre ambos términos, el término emoción a menudo es usado para referirse sólo al estado corporal (estado emocional, como por ejemplo una alteración en la frecuencia cardíaca y respiratoria, la contracción y relajación involuntaria de los músculos faciales y la emisión de sonidos), mientras que el término sentimiento se refiere únicamente a una sensación concreta (como pueden ser el miedo, la alegría, la ira, la tristeza, el placer o la ansiedad). El estado emocional y los sentimientos son regulados por estructuras anatómicas diferentes. De esta forma, los sentimientos concretos están regulados por la corteza cerebral, en parte por la corteza cingulada y la corteza orbitofrontal. Los estados emocionales están regulados por un conjunto de respuestas periféricas, autónomas, endocrinas y esquelético-motoras.

Estas respuestas involucran estructuras subcorticales, tales como la amígdala, el hipotálamo y el tallo cerebral. Ante la sensación de terror, no sólo se siente miedo sino que también se experimenta un aumento en la frecuencia cardiaca y respiratoria, la boca se seca, se tensan los músculos, sudan las palmas de las manos (Carey, Ariniello, \& McComb, 2002).

Para entender una emoción como el miedo, es necesario entender primero la relación entre el sentimiento cognitivo representado en la corteza cerebral y los signos fisiológicos asociados regulados por las áreas subcorticales (Kandel, 2000).

Siguiendo este orden de ideas, un estímulo emocional con una intensidad significativa activa sistemas sensoriales que envían la información hacia el hipotálamo, el cual genera una respuesta capaz de modular la frecuencia cardiaca, la tensión arterial y la frecuencia respiratoria. Al mismo tiempo, la información de este estímulo es llevada hasta la corteza cerebral, de modo 
que el estímulo y la información son llevados indirectamente desde los órganos periféricos (los cuales perdieron su estado homeostático debido al estímulo) y directamente desde el hipotálamo, la amígdala y las estructuras relacionadas. De manera simultanea, la información del estímulo se lleva a la corteza cerebral, indirectamente desde los órganos periféricos (los cuales, perdieron su estado homeostático debido al estímulo) y directamente desde el hipotálamo, la amígdala y estructuras relacionadas (Purves, 2004).

En 1883, James Papez (1929) propuso que la estructura de origen cortical que se encarga de representar y determinar los sentimientos es el lóbulo límbico. Esta estructura fue identificada previamente por Paul Broca. El término de sistema límbico fue acuñado por Paul McLean, quien lo consideró así debido a que se incluían partes del hipotálamo, el área septal, el núcleo accumbens, las áreas neocorticales y la amígdala (Figura 2A).

El lóbulo límbico es un anillo filogenéticamente proveniente de corteza primitiva que se encuentra sobre el tallo cerebral e incluye el giro cingulado, el giro parahipocampal y la formación hipocampal, que se localiza en la parte profunda del giro parahipocampal. Su estructura morfológica es tan simple como una corteza enrollada. La formación hipocampal incluye al hipocampo, al giro dentado y al subínculo (Roffman, Marci, Glick, Dougherty \& Rauch, 2005).

Papez (1929) señala que la comunicación entre el hipocampo y la corteza cerebral (neocorteza) se lleva a cabo de manera recíproca. De esta forma, la neocorteza y el hipotálamo están en comunicación constante y una estructura influye en la otra por medio del giro cingulado. Así, la comunicación se llevaría en el siguiente orden: la formación hipocampal procesa la información que proviene del giro cingulado y la lleva hasta los cuerpos mamilares del hipotálamo vía fórnix (fibras que en parte sacan información del hipocampo). Al mismo tiempo, el hipotálamo envía información al giro cingulado por la vía cuerpos mamilares-núcleo talámico anterior (tracto mamilo-talámico) y de aquí a la corteza frontal (Figura 2B).

Históricamente hablando, la primera evidencia que relacionó al sistema límbico con las emociones se tiene registrada en 1955, cuando Heinrich Klüver y Paul Bucy describieron un síndrome conductual inducido
Figura 2. Esquema que muestra la relación anatómica de cada una de las estructuras del sistema límbico en el humano.

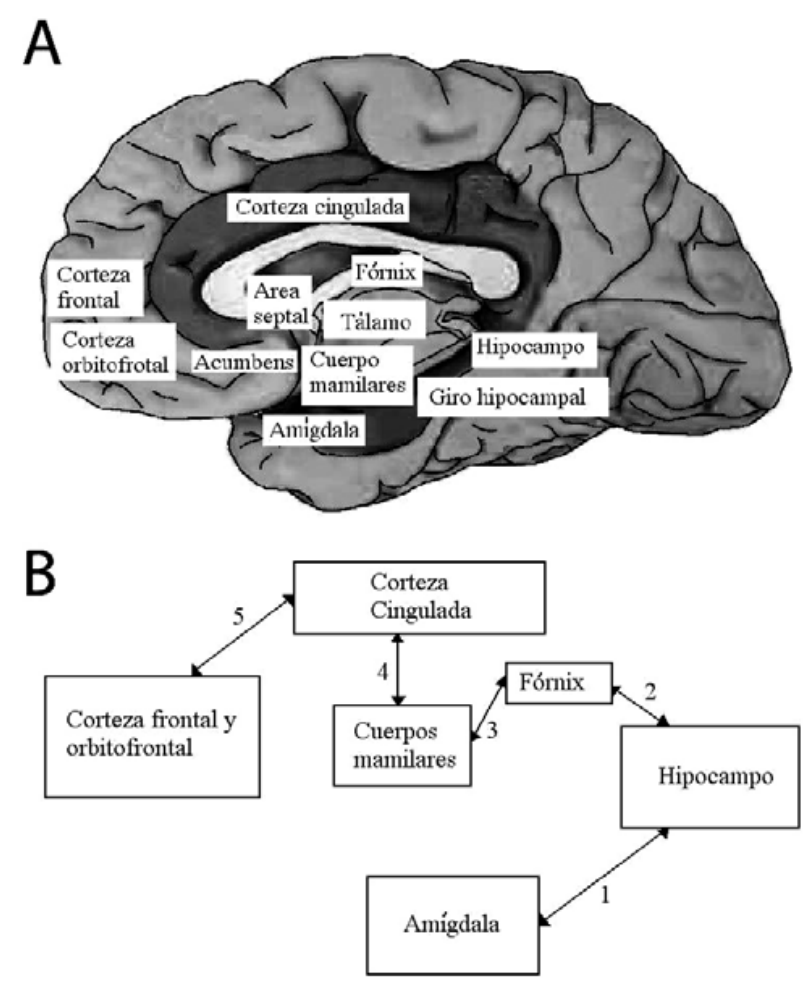

Nota: A Disposición anatómica del sistema límbico en el humano. B Flujo de señales que se establece entre las estructuras del sistema límbico, lo que determina una cadena neuronal que representa la base fisiológica del sistema que regula las emociones.

en el laboratorio, el cual incluía un cambio sustancial en la conducta emocional. En sus experimentos observaron que los monos, que eran tranquilos en extremo, sufrían cambios emocionales como: agresividad y pérdida del miedo luego de ser sometidos a una lobotomía bilateral de los lóbulos temporales, la cual también incluyó la amígdala, la formación parahipocampal y una estructura hasta el momento no considerada como estructura límbica: la corteza temporal. Estos monos presentaron también otros cambios conductuales como un incremento en la conducta sexual, que se manifestaba en que montaban objetos y especies inapropiadas y en una conducta obsesiva para observar y reaccionar ante cada estímulo visual; sin embargo, no eran capaces de reconocer los objetos familiares (Bucy \& Klüver, 1955). 
Diversos experimentos en humanos y en animales permitieron posteriormente establecer que la amígdala, que intercomunica al sistema de la expresión somática de las emociones (hipotálamo y núcleos del tallo cerebral), es el sistema de los sentimientos concisos, especialmente el miedo (cortezas cingulada, parahipocampal y frontal (Figura 2).

Cuando se estimula eléctricamente la actividad de la amígdala a través de experimentos, en los humanos se produce miedo y aprehensión (Lanteaume, Khalfa, Régis, Marquis, Chauvel \& Bartolomei, 2007). En los animales, quitar la amígdala genera una conducta de ausencia de miedo. En la práctica neurológica clínica se ha descrito la enfermedad de Urbach-Wiethe, que es una enfermedad neurodegenerativa asociada con un depósito de calcio en la amígdala, genera una pérdida de la actividad de la amígdala de manera bilateral, lo que se traduce en una conducta incapaz de reconocer claves en la expresión de la cara de las personas que denotan miedo. De la misma forma, estas personas pierden la habilidad para discriminar pequeñas diferencias en la expresión facial de las personas. Esta enfermedad inhibe el procesamiento de las claves emocionales que se pueden distinguir al observar la cara de las personas que actúan o realmente experimentan miedo (Siebert, Markowitsch \& Bartel, 2003). Estos pacientes no pierden la habilidad de reconocer caras familiares aun cuando haya pasado mucho tiempo de haberlas visto. Lo anterior indica que son dos los sistemas necesarios para, por un lado reconocer la identidad de las personas y por otro las características de su expresión facial. Estas estructuras indudablemente son la amígdala y la corteza visual (Siebert et al., 2003).

Desde el punto de vista evolutivo y anatómico, el cerebro de los mamíferos relacionados con el del Homo sapiens, como son: los monos, el gibón, el gorila, el orangután y el chimpancé, presenta estructuras cerebrales similares a las que forman lo que hoy conocemos como el sistema límbico en el humano. Las principales son una pequeña área de corteza frontal, el sistema límbico, el hipotálamo, la amígdala y el hipocampo. Mediante un gran número de paradigmas experimentales básicos y conductuales se ha mostrado la funcionalidad equiparable de estas estructuras cerebrales de los primates con la conducta y fisiología neuronal del humano. Por ejemplo, en los monos rhesus in- fantes y adultos, los estímulos sensoriales que producen una respuesta emocional de miedo (que puede ser desencadenado por al aislar a los bebés de sus madres) pueden clasificarse en tres tipos de conductas bien caracterizadas: cooing (emisión de un sonido similar al "coo"), freezing (una conducta de congelamiento o de falta de movimiento asociado a la exposición temeraria de los dientes) y barking (conducta agresiva con la emisión de sonidos amenazantes) (Kalin \& Shelton, 1989; Kalin, Shelton, Davidson \& Kelley, 2001).

Otros animales también comparten algunas características de anatomía y fisiología del sistema límbico humano en cuanto a la expresión de las emociones. Las ratas, por ejemplo, también generan conductas específicas ante estímulos sensoriales que evocan respuestas emocionales. La incidencia de haces de luz en sus sitios predilectos para dormir, o la aplicación de choques eléctricos en sus patas, genera en ellas conductas emocionales de miedo que han sido bien caracterizadas (por ejemplo: freezing, piloerección e incremento en la defecación). Estas conductas han sido cuantificadas y analizadas farmacológicamente para determinar la potencialidad de ciertos fármacos para el tratamiento del estrés y la ansiedad, conductas involucradas en el proceso de la expresión de las emociones.

\section{EMPATÍA}

La capacidad de generar empatía en los animales, así como sucede con los humanos, ha sido motivo de una gran cantidad de estudios, en los cuales se incluyen roedores, primates e incluso humanos. Hay que tomar en cuenta que en la empatía debe existir primero una conexión emocional seguida de la comprensión y de la respuesta motora que involucre un compromiso con los sentimientos de la otra persona. En un estudio reciente realizado en la Universidad de McGill en Canadá, consistió en aplicar choques eléctricos a pares de ratones que habían habitado la misma caja de almacenaje por lo menos diez días; los resultados permitieron ver cuál de los dos ratones mostró menos dolor al estímulo (es decir, al choque eléctrico), y al mismo tiempo cuál ratón mostró más dolor a pesar de presentarle el estímulo (el choque eléctrico) sin cambio en la intensidad del mismo. De igual manera, al cambiar el estímulo doloroso por una inyección peritoneal de una solución diluida de acido acético (la cual produce dolor 
en el estomago y genera una conducta de estiramiento de los ratones), el segundo ratón presentó más dolor en vista de que tuvo más movimientos de estiramiento, comparado con el primero. Este efecto sólo se observó en ratones pareja y en mayor proporción en ratones macho. En ratones macho que no se conocían, este efecto no se presentó, posiblemente por la conducta innata de rivalidad en estos animales (Langford et al., 2006). Estos resultados sugieren que para que se inicie el proceso de empatía debe haber primero una identidad emocional, la cual está generada principalmente por el conocimiento y entendimiento de las emociones del otro animal, específicamente en este experimento. Cuando esta conexión no existe, la empatía se traduce en antipatía.

Un experimento clave en el proceso de la generación de la empatía fue el realizado por Giacomo Rizzolatti en 1996, de la Universidad de Parma, en Italia. Consistió en registrar neuronas premotoras de la corteza prefrontal en monos rhesus y observar qué sucede cuando el mono toma un cacahuate para comérselo. Cuando el mono realiza esta acción, la neurona motora de la corteza dispara potenciales de acción con una frecuencia característica. Esta actividad eléctrica de las neuronas de la corteza prefrontal no aparece cuando el mono sólo toma el cacahuate y lo coloca en un recipiente. A la actividad de las neuronas premotoras se le conoce como actividad "tomacome". Cuando el mono observa a un humano comer un cacahuate, la neurona premotora se activa; pero, cuando éste observa que el humano sólo toma el cacahuate y lo coloca en un recipiente, la neurona no se activa (Iacoboni, Molnar-Szakacs, Gallese, Buccino, Mazziotta \& Rizzolatti, 2005). Éste es el principio básico para la descripción de las neuronas espejo que también están involucradas en el proceso de generación de empatía en humanos y animales.

Por último, un estudio realizado por Tania Singer y colegas (Singer, Seymour, O'Doherty, Stephan, Raymond, Dolan, et al., (2006) de la Universidad de Zurich realizaron un estudio dónde muestran la importancia del género de las especies en la manifestación de la empatía. Parejas de hombres y mujeres fueron sometidos a un estudio en el que uno a la vez aplicaba un estímulo doloroso a su compañero. Mientras, por medio del análisis de la Imagen de Resonancia Magnética funcio- nal (MRIf), se observaba la actividad del cerebro en uno de ellos cuando observan el dolor de su pareja. Las áreas cerebrales relacionadas con el dolor se activan de inmediato, en mayor medida en los hombres que en las mujeres. Sin embargo, cuando se colocan parejas de hombres, invariablemente ambos sienten que han sido tratados de modo injusto por el otro hombre en la prueba de dolor previa, lo que induce que sus centros de placer cerebrales se activen cuando observa al hombre experimentar dolor. Las áreas cerebrales relacionadas con el dolor se activan inmediatamente, en mayor medida en los hombres comparados con las mujeres. Sin embargo, cuando se colocan parejas de hombres, que previamente se aplicaban uno al otro estímulos dolorosos y posteriormente se analizaba su actividad cerebral mientras observaban el dolor de su compañero, éstos muestran una mayor actividad en sus centros de placer, es decir el núcleo accumbens se activa cuando se observa a otro (hombre o persona) experimentar dolor. Cuando este observador ha sido también tratado previamente con dolor, en la situación de de ver "sufrir" al primero, en el segundo, su cerebro produce una sensación de placer. Dicha respuesta no es otra cosa, como se puede ver, que una muestra o expresión de un sentimiento de antipatía, misma que en el hombre se interpreta como de rivalidad, respuesta típica en mamíferos según Singer et al. (2006).

Otro de los resultados interesantes de este estudio fue que el núcleo accumbens se activa más cuando existía más sentimiento de revancha. La activación de esta estructura y el sentimiento de revancha muestran una correlación estadísticamente significativa, la cual es mayor en los hombres que en las mujeres (Figura 3).

\section{GENERADORES DE PATRONES CENTRALES (CPG'S) Y EMOCIONES}

Los CPG's inicialmente descritos en animales sencillos como la lamprea han sido estudiados y descritos en algunas de las conductas humanas (Grillner, Cangiano, $\mathrm{Hu}$, Thompson, Hill \& Wallen, 2000; Grillner \& Wallen, 2002). Por ejemplo, se ha descrito la presencia de CPG's en la expresión facial de las emociones. Cuando un estímulo externo provoca una emoción en los animales y en el hombre, varios núcleos cerebrales se comunican entre sí, los cuales finalmente activan la contracción y relajación de ciertos músculos faciales. Desde el punto 
Figura 3.

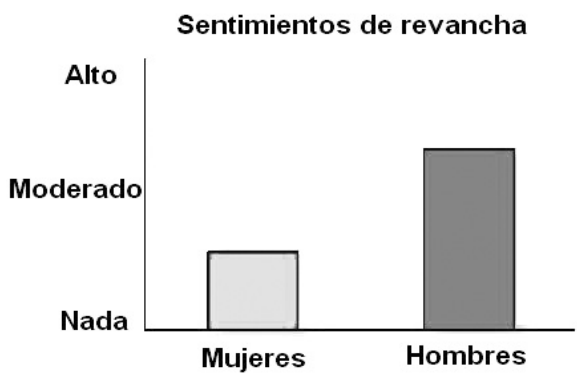

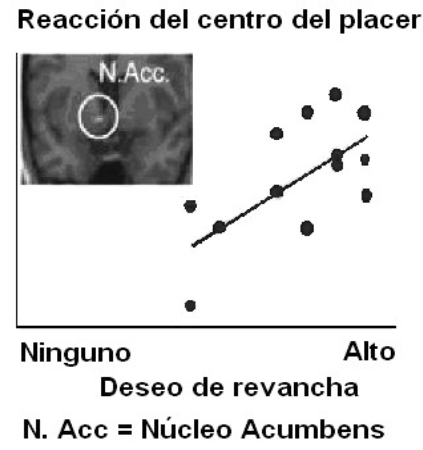

Nota: Gráficas que muestran la cuantificación del sentimiento de revancha, el cual se obtuvo por medio de una prueba psicológica. De esta gráfica se concluye que el hombre tiene un sentimiento de revancha más alto. La conducta observable es la de aplicar un estímulo mas grande para resarcir el dolor que éste sufrió previamente. En la segunda gráfica se observa que el sentimiento de revancha en hombres está relacionado con un deseo de revancha que se asocia al aumento en la actividad del núcleo acumbens, el cual puede observarse en el recuadro. de vista neuroanatómico, el CPG estaría formado por la corteza motora y los músculos motores, si se trata de una respuesta emocional voluntaria. Sin embargo, este circuito es diferente si se trata de una respuesta emocional involuntaria, el cual incluye al sistema límbico, la corteza motora y los músculos faciales. Reportes clínicos de ciertas enfermedades neurológicas (parálisis facial) apoyan la distinción entre expresiones faciales voluntarias e involuntarias (Danner, 2008). Cada una de estas expresiones faciales depende de rutas neuronales diferentes e independientes. Por ejemplo, lesiones en el sistema piramidal impiden la ejecución de movimientos faciales cuando éstos son solicitados, tales como sonreír. Sin embargo, cuando al paciente se le relata un chiste, éste puede sonreír de manera involuntaria (Michel, Derkinderen, Laplaud, DaumasDuport, Auffray-Calvier \& Lebouvier, 2008). Lesiones en el sistema no piramidal inducen la conducta clínica opuesta: el paciente sonríe cuando se le solicita, pero no cuando es involuntaria la conducta (cuando se le relata un chiste).

La disección del CPG involucrado en la risa de los humanos fue en primera instancia abordada por Duchenne de Bologne en 1990, cuando estudió la diferencia entre la risa de las personas asociada o no a la felicidad. Duchenne comparó la risa de un sujeto inducida por la estimulación eléctrica del músculo mayor zigomático (respuesta eléctrica "voluntaria”), con la risa provocada por un chiste (respuesta "involuntaria") en el mismo sujeto. La respuesta involuntaria no sólo incluye la actividad del músculo mayor zigomático, sino también la actividad del músculo ocular orbicularis, el cual rodea al ojo y jala las mejillas hacia arriba, produce arrugas en la esquina externa del ojo y baja ligeramente las cejas. Desde esa época, Duchenne, concluyó que” [...] la persona que no tuviera este patrón en su cara, no era un amigo verdadero" (Duchenne De Boulogne, 1990). Como se había comentado con anterioridad, estos elementos son claves para el reconocimiento y entendimiento de las emociones y necesarios para la generación de empatía. Evidentemente, en la sonrisa involuntaria está involucrado el sistema límbico en el CPG's, que contiene: a la corteza motora, al músculo mayor zigomático y al músculo ocular orbicularis.

En los primates, que también cuentan con músculos faciales, estos CPG's han sido ampliamente estudiados y están involucrados con las conductas sociales importantes para la participación de los individuos en grupo. El elemento diferente en los CPG's de los primates es la co-evolución de la neocorteza, la cual presenta menor desarrollo en su parte frontal y temporal comparada con el cerebro humano. Estas diferencias anatómicas provocan diferencias conductuales sociales. Sin embargo, la preservación de los CPG's (sistema límbico, músculos faciales: mayor zigomático, ocular orbicularis y oris orbicularis) representa desde el punto de vista evolutivo la importancia de la expresión de las emociones y la generación de empatía para el desarrollo individual y del grupo social al que pertenecen los animales. En otras especies animales, estos CPG's incluyen predominantemente músculos de otras partes del cuerpo para denotar la expresión de las emociones; por ejemplo, en los roedores, los gatos y los perros, la piloerección, mostrar los dientes y/o colmillos, y adoptar 
una posición corporal de supremacía es vital. La empatía que estos signos generan en los demás animales es la de denotar peligro, compañía, alegría e incluso repulsión (Figura 4).

Figura 4.

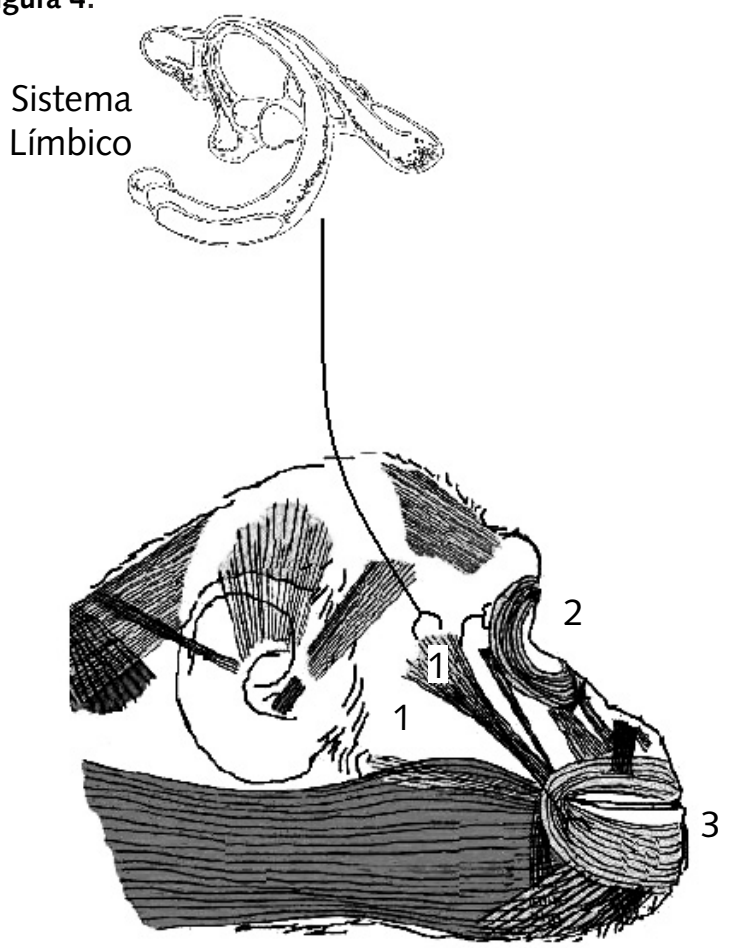

Nota: Circuito neuronal que representaría un CPG en los primates, el cual controla el movimiento de los músculos faciales para generar una señal fisiológica conocida como emoción gestual, la cual alertará a los demás animales de su sociedad. 1 Músculo zigomático mayor, 2 Músculo ocular orbital y 3 Músculo oral orbital.

\section{CONCLUSIÓN}

De los experimentos realizados en animales, sobre todo en ratas y primates, si bien no es posible hablar estrictamente de que se generen sentimientos durante estos experimentos, es indudable que se demuestra que se genera en ellos un cambio en la intensidad de sus experiencias. Esta demostración nos permite hablar de la empatía no sólo en términos humanos sino también en el reino animal, por lo menos en los animales que muestran un cerebro evolucionado. En términos científicos, existen dos corrientes que observan el mismo fenómeno desde dos puntos de vista totalmente dife- rentes. Por un lado están los psicólogos, que consideran a la empatía como una de las formas más avanzadas de la conducta humana, algo así como colocarse en los zapatos de otra persona e imaginar su situación. Este proceso sin duda requiere de ciertas habilidades, como una avanzada capacidad de razonamiento e incluso el lenguaje. Sin embargo, el simple hecho de imaginar lo que alguien siente, no se considera empatía. Ésta requiere una conducta cognoscitiva y de relación emocional. Por otro lado, los biólogos de la conducta y los neurocientíficos observan el mismo fenómeno y consideran que cuando reaccionamos observando la respuesta emocional de otra persona, y construimos un razonamiento de su situación, involucramos a otras estructuras anatómicas que forman parte de lo que hoy conocemos como el sistema límbico.

Este proceso, según observaciones en humanos y en animales experimentales, señala que, como primer paso, es indispensable el involucramiento emocional para después generar el entendimiento de lo que está sucediendo y de la emoción percibida, y finalmente "imaginarse" esta respuesta en el contexto que está sucediendo. La respuesta observada en animales experimentales sugiere fuertemente que este componente emocional (empatía o antipatía) es un proceso viejo y se ha conservado en nosotros desde el ancestro de los mamíferos hasta hoy, formando una parte crucial de la conducta de los humanos.

La presencia de las estructuras involucradas en las respuestas emocionales a lo largo de la escala filogenética, así como en la escala evolutiva del sistema nervioso central en los mamíferos, apoya la idea de que el proceso de neurogénesis y migración neuronal favorece la expresión y formación de redes anatómicamente funcionales, que están encaminadas a regular ciertas conductas inherentes al proceso evolutivo y preservativo de la especie. Estas redes neuronales son la que han recibido recientemente el nombre de CPG's, y son las que regulan conductas estereotipadas en los mamíferos. Estos centros pueden enriquecerse y generar respuestas motoras o conductas tan refinadas y específicas en función directa a las experiencias previas y al entorno que rodea al hombre y a los animales.

Estos CPG's permitirían también la expresión objetiva de las emociones, la cual involucraría el movimiento de ciertos músculos faciales, entre otras. Tales 
músculos, dependiendo del estímulo sensorial, tendrían socialmente hablando la función de alertar a los sujetos que le rodean sobre la naturaleza del estímulo que dio origen a esa conducta. De esta forma, nuestro cerebro estaría capacitado para discernir entre los diferentes signos que denotan los movimientos faciales

\section{REFERENCIAS}

Barton, R. A., Aggleton, J. P. \& Grenyer, R. (2003). Evolutionary coherence of the mammalian amygdale. Proceedings of the Royal Society of London, 270, 539-543.

Briggman, K. L. \& Kristan, W. B. (2006). Imaging Dedicated and Multifunctional Neural Circuits Generating Distinct Behaviors. Journal of Neuroscience. 26, 42, 10925-10933.

Bucy, P. C. \& Klüver, H. (1955). An anatomical investigation of the temporal lobe in the monkey (Macaca mulatta). The Journal of comparative neurology, 2, 103, 151-251.

Carey, J., Ariniello, L. \& McComb, M. (2002). Brain Facts. A primer on the Brain and Nervous System. Washington, DC, USA. Science for Neuroscience Press.

Danner, C.J. (2008). Facial nerve paralysis. Otolaryngologic clinics of North America, 41, 3, 619-632.

Darwin, C. (1998). The Expression of the Emotions in Man and Animals, $3^{\text {rd }}$ edit. Introduction, afterwords, and commentaries by Paul Ekman. Harper Collins. London. Nueva York: Oxford University Press.

Duchenne De Boulogne, G.B. (1990). The Mechanism of Human Facial Expression. Nueva York: A. Cuthbertson, Trans. \& Ed. Cambridge University Press.

Gelder, B. de, Morris, J. S., \& Dolan, R. J.(2005). Unconscious fear influences emotional awareness of faces and voices. Proceedings of the National Academy of Sciences of the United States of America, 102, 55, 18682-18687.

Giacomo, R. (1996). "Somos grandes copiadores, sin imitación no habría cultura”. Artículo Eleéctronio recuperado el 9 de noviembre de 2009 de http:// www.publico.es/ciencias/142076/somos/grandes/copiadores/imitacion/cultura/giacomo/rizzolatti/neurobiologo/aprendizaje.

Grillner, S., Cangiano, L., Hu, G., Thompson, R., Hill, R. para generar una conducta de empatía, la cual es importantísima para el humano que vive en sociedad, así como para los animales que viven en una estructura social que les involucra en actividades de defensa y cooperación.

\& Wallen, P. (2000). The intrinsic function of a motor system - from ion channels to networks and behavior. Brain Research, 886, 224-236.

Grillner, S. \& Wallen, P. (2002). Cellular bases of a vertebrate locomotor system-steering, intersegmental and segmental co-ordination and sensory control. Brain Research Reviews, 40, 92-106.

Heltne, P. \& Marquardt, L. A. (1989). Understanding Chimpanzees. USA: Harvard University Press.

Iacoboni, M., Molnar-Szakacs, I., Gallese, V., Buccino, G., Mazziotta, J. C. \& Rizzolatti, G. (2005). Grasping the Intentions of Others with One's Own Mirror Neuron System. Plos Biology, 3, 3, 529-535.

Kalin, N. H. \& Shelton, S. E. (1989). Defensive Behaviors in Infant Rhesus Monkeys: Environmental Cues and Neurochemical Regulation. Science, 243, 1718-1721.

Kalin, N. H., Shelton, S. E., Davidson, R.J. \& Kelley, A. E. (2001). The Primate Amygdala Mediates Acute Fear but Not the Behavioral and Physiological Components of Anxious Temperament. Journal of Neuroscience, $21,6,2067-2074$.

Kandel, E. (2000). Principles of Neural science. New York, USA: McGraw Hill. Korb S., Grandjean, D. \& Scherer, K. (2008). Motor Commands of Facial Expressions: The Bereitschafts potential of Posed Smiles. Brain topography, 20, 232-238.

Korb, S., Grandjean, D. \& Scherer, K. (2008). Motor Commands of Facial Expressions: the Bereitschaftspotential of Posed Smiles. Brain Topography, 20, 4, 232-238.

Langford, D. J., Crager, S. E., Shehzad, Z., Smith, S. B., Sotocinal, S. G., Levenstadt, J. S., Chanda, M. L., Levitin, D. J. \& Mogil, J. S. (2006). Social Modulation of Pain as Evidence for Empathy in Mice. Science, 312, 5782, 1967-1970. 
Lanteaume, L., Khalfa, S., Régis, J., Marquis, P., Chauvel, P. \& Bartolomei, F. (2007). Emotion induction after direct intracerebral stimulations of human amygdala. Cerebral Cortex, 17, 6, 1307-1313.

Lovecky, D. V. (2004). Different minds: Gifted children with $A D H D$, Asperger Syndrome and others learning deficits. London, England: Jessica Kingsley Publishers.

Michel, L., Derkinderen, P., Laplaud, D., Daumas-Duport, B., Auffray-Calvier, E. \& Lebouvier, T. (2008). Emotional facial palsy following striato-capsular infarction. Journal of neurology, neurosurgery, and psychiatry, 79, 2, 193-194.

Papez, J. W. (1929). Comparative neurology; a manual and text for the study of the nervous system of vertebrates. New York, Thomas Y. Crowell company.

Purves, D. (2004). Neuroscience. Sunderland, Massachusetts, USA: Sinauer Associates Inc.
Randall L. S., (1984). The Pygmy Chimpanzee: Evolutionary Biology and Behavior. Nueva York, USA. Plenum Press.

Roffman, J. L., Marci, C. D., Glick, D. M., Dougherty, D. D. \& Rauch, S. L. (2005). Neuroimaging and the functional neuroanatomy of psychotherapy. Psychological Medicine, 35, 1-14.

Siebert, M., Markowitsch, H.J. \& Bartel, P. (2003). Amygdala, affect and cognition: evidence from 10 patients with Urbach-Wiethe disease. Brain, 126, 2627-2637.

Singer, T., Seymour, B., O’Doherty, J. P., Stephan, K. L., Raymond, J., Dolan, R. J. \& Frith, C. D. (2006). Empathetic Neural Responses Are Modulated by the Perceived Fairness of Others. Nature, 439, 466-469. 